\title{
Burden of Infectious Disease in a State of India: A Comparative Analysis
}

\section{Divya Persai*}

Public Health Foundation of India, New Delhi, India

\section{Objective}

The present study aims to analyze data from the Integrated Disease Surveillance Project (ISDP) to estimate the rates of selected infectious diseases in the state of Maharashtra in comparison to the rest of India.

\section{Introduction}

In India the range and burden of infectious diseases is enormous. To address this burden effectively, an estimate of the burden of infectious diseases is essential. The present study aims to analyze data from the Integrated Disease Surveillance Project (ISDP) to estimate the rates of selected infectious diseases in the state of Maharashtra in comparison to the rest of India.

\section{Methods}

District-wise and disease-wise disaggregated data from 20052012 was collated. Annual rates was calculated and analysis of epidemiological profile, including spatial trends, rural-urban distributions and annual case rates was done. A comparison of Maharashtra IDSP case rates with case rates from the national data was made. Data was analysed using SPSS 17.

\section{Results}

Findings indicate that the existing surveillance system in the state is predominantly reporting urban cases. There are wide variations among reported cases within the state with reports of Enteric fever and Measles, Cholera, Viral Hepatitis and Dengue.

\section{Conclusions}

The present study shows that the existing surveillance system in the state is predominantly reporting urban cases. There is a need to strengthen IDSP into a comprehensive surveillance system in order to reverse the endemicity of these diseases.

\section{Keywords}

Disease Survelliance; India; Infectious Disease

\section{References}

1.John J, Dandona L, Sharma V, Kakkar M. Continuing challenge of infectious diseases in India. The Lancet, 377: 252 - 269; 2011.

\section{*Divya Persai}

E-mail: dpersai@gmail.com 